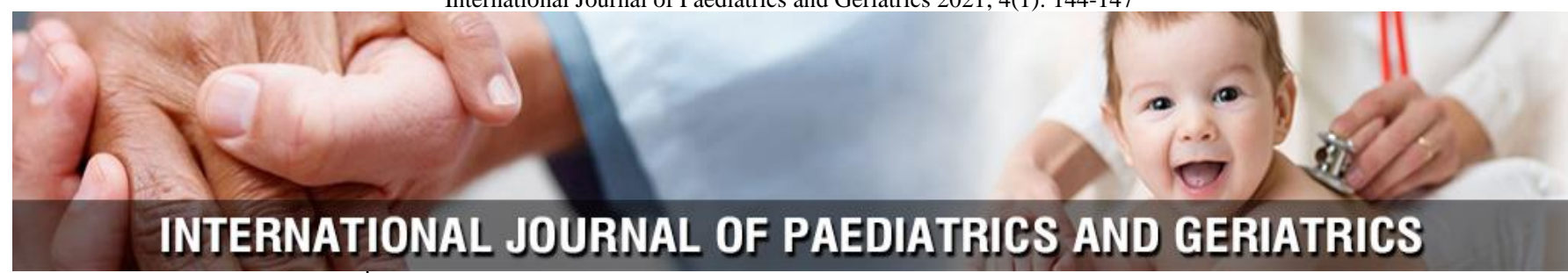

P-ISSN: 2664-3685

E-ISSN: 2664-3693

www.paediatricjournal.com

IJPG 2021; 4(1): 144-147

Received: 16-02-2021

Accepted: 18-04-2021

\section{Sofia Ferrito}

Department of Pediatrics,

Hospital Garcia de Orta,

Avenida Torrado da Silva,

Almada, Portugal

\section{Rita Marques}

Department of Pediatrics,

Hospital Garcia de Orta,

Avenida Torrado da Silva,

Almada, Portugal

\section{Maria Cabral}

Department of Pediatric

Surgery, Hospital Garcia de

Orta, Avenida Torrado da

Silva, Almada, Portugal

\section{Filipa Nunes}

Department of Pediatrics, Hospital Garcia de Orta.

Avenida Torrado da Silva, Almada, Portugal

\section{Margarida Pinto}

Department of Pediatrics, Hospital Garcia de Orta,

Avenida Torrado da Silva,

Almada, Portugal
Corresponding Author: Sofia Ferrito

Department of Pediatrics, Hospital Garcia de Orta, Avenida Torrado da Silva, Almada, Portugal

\section{Peritoneal and genital tuberculosis in an adolescent: A challenging diagnosis}

\author{
Sofia Ferrito, Rita Marques, Maria Cabral, Filipa Nunes, Margarida \\ Pinto
}

DOI: $\underline{\text { https://doi.org/10.33545/26643685.2021.v4.i1c.145 }}$

\begin{abstract}
Introduction: Extra-pulmonary manifestations of tuberculosis occur in about $20 \%$ of cases. Peritoneal and genital tuberculosis are uncommon, especially in children without any comorbidity.

Case report: A previously healthy 14-year-old girl, presented complaints of weight loss, anorexia, abdominal pain and fever. On examination, she was febrile, with rebound abdominal tenderness and abdominal distension. Ultrasonography reported peritoneal fluid, fibrin strands and complex cystic lesions in the adnexal region. Laparotomy evidenced multiple peritoneal cysts, purulent exudate with bilateral pyosalpinx. Mycobacterium tuberculosis was isolated in culture and confirmed the diagnosis of Peritoneal Tuberculosis. Antituberculous quadruple therapy and corticosteroids were started, with good clinical response.

Conclusions: Peritoneal and genital tuberculosis are uncommon in children, but have high fatality and infertility rate. Due to the nonspecific clinical, laboratory or radiology findings, diagnosis is often delayed. This report highlights the need for a big suspicious index of the disease for an accurate and early diagnosis.
\end{abstract}

Keywords: peritoneal, genital, tuberculosis, adolescent, children, corticosteroids

\section{Introduction}

Tuberculosis (TB) continues to be a major health problem throughout the world. Coinfection with human immunodeficiency virus (HIV), more liberal immigration from high risk to low risk areas due to globalization, multidrug resistant and poverty conditions are important contributors ${ }^{[1,2]}$. Approximately one third of the world's population is infected with Mycobacterium tuberculosis (Mt) ${ }^{[1]}$. Pulmonary TB constitutes the more common clinical presentation of TB ${ }^{[3]}$. Extra-pulmonary manifestations of TB occur in about $20 \%$ of cases ${ }^{[1]}$. Abdominal TB is rare in children without any comorbidity such as cirrhosis, HIV infection, diabetes mellitus, cancer, treatment with anti-tumor necrosis factor agents and use of peritoneal dialysis ${ }^{[1,4]}$, with a similar prevalence across all paediatric age groups and sexes ${ }^{[1,5]}$. In adults, $3 \%$ of TB cases have abdominal involvement compared with $0.3 \%$ of paediatric cases ${ }^{[5]}$. Abdominal tuberculosis, i.e. infection of the gastrointestinal tract, the mesentery, its nodes, omentum, peritoneum, and related solid organs, presents in a clinical spectrum depending on the site of involvement ${ }^{[6]}$. Peritoneal TB involves insidious onset and symptoms are non-specific: abdominal pain, fever, diarrhea and constipation, weight loss, anorexia, abdominal distension, ascites and malaise ${ }^{[1,7]}$. It can be classified into three types: a wet type characterised by significant ascites, a dry type with adhesions and a fibrotic type with omental thickening and loculated ascites ${ }^{[5,8]}$. When TB affects the genital organs of young females, it has the devastating effect of causing irreversible damage to the fallopian tubes, resulting in infertility difficult to cure by medical and surgical methods [7]. Pathogenesis involves infection by tubercle bacilli following reactivation via hematogenous spread from a primary lung focus, via hematogenous spread from active pulmonary or miliary TB, and through lymph channels from infected nodes. Peritoneal involvement may occur from infected contiguous lymph nodes, intestinal lesions, or fallopian tubes in women $[1,8]$. Owing to the non-specific clinical manifestations, diagnosis of abdominal TB is often delayed, resulting in higher rates of morbidity and mortality, particularly in areas of low TB incidence ${ }^{[5]}$. Peritoneal TB is a rare entity in the literature and will be discussed in this case report. 


\section{Case report}

A previously healthy 14-year-old girl, presented to the emergency department with a 1-month history of unintentional weight loss and anorexia associated with abdominal pain and fever for the last week. She denied any respiratory symptoms, night sweats, sexually active life or abnormal vaginal discharge. She lived in Angola up until 6 months and her mother had a past medical history of treated pulmonary TB. On physical examination, she presented with $38.1{ }^{\circ} \mathrm{C}$ body temperature, pale mucous membranes, moderate abdominal distension and rebound lower abdominal tenderness. There was a bacillus CalmetteGuerin (BCG) scar on her left upper arm. Laboratory results revealed a hypochromic microcytic anaemia (hemoglobin $7.0 \mathrm{~g} / \mathrm{dL})$, normal white blood cell count $\left(10.0 \times 10^{9} / \mathrm{L}\right)$, thrombocytosis $\left(608000 \times 10^{9} / \mathrm{L}\right)$, elevated C-reactive protein level $(10.8 \mathrm{mg} / \mathrm{dL})$, erythrocyte sedimentation rate (120 mm/hour) and lactate dehydrogenase (500 UI/L). Liver and kidney function tests were normal. HIV 1 and 2, Syphilis, hepatitis B and C serologies were negative. There were no pathological findings on her chest radiography. Abdominal ultrasonography and computed tomography reported a large amount of peritoneal fluid, fibrin strands and complex cystic lesions in the adnexal region. Exploratory laparotomy evidenced multiple peritoneal cysts, abundant purulent exudate and frozen pelvis with bilateral pyosalpinx. Drainage and biopsies were performed. Differential diagnosis between Pelvic Inflammatory Disease and Peritoneal Tuberculosis (PTB) were considered. Intavenous antibiotics (ampicillin, gentamicin and metronidazole) were started. A Mantoux tuberculin skin test was positive resulting in an induration with a diameter of 23 $\mathrm{mm}$ after 72 h. Histology showed granulomatous inflammation with giant multinucleated cells with central necrosis. Ziehl-Neelsen staining of the ascitic fluid was negative. Mycobacterium tuberculosis was isolated in culture and confirmed the diagnosis of Peritoneal Tuberculosis. Tuberculostatic treatment was initiated on quadruple therapy with isoniazid, rifampicin, pyrazinamide and ethambutol for 2 months followed by 4 months of dual therapy with isoniazid and rifampicin. Corticosteroids (prednisolone $1.5 \mathrm{mg} / \mathrm{kg} /$ day) were administered for the first month of therapy. She had good clinical and laboratorial response after 6 months of treatment, with ascites and associated symptoms resolved. A 3 years follow-up showed no TB recurrence or treatment-related complications.

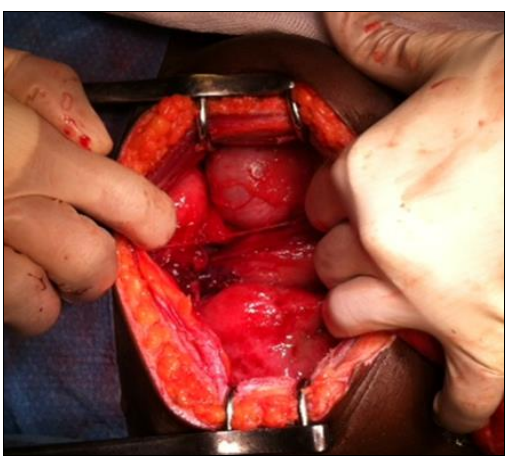

A

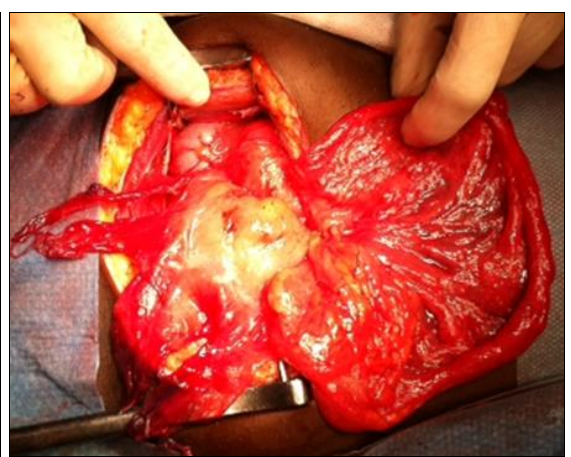

B

Fig 1, 2: Laparotomy findings showing multiple peritoneal cysts (A), abundant purulent exudate (B) and frozen pelvis with bilateral pyosalpinx $(\mathrm{A}, \mathrm{B})$

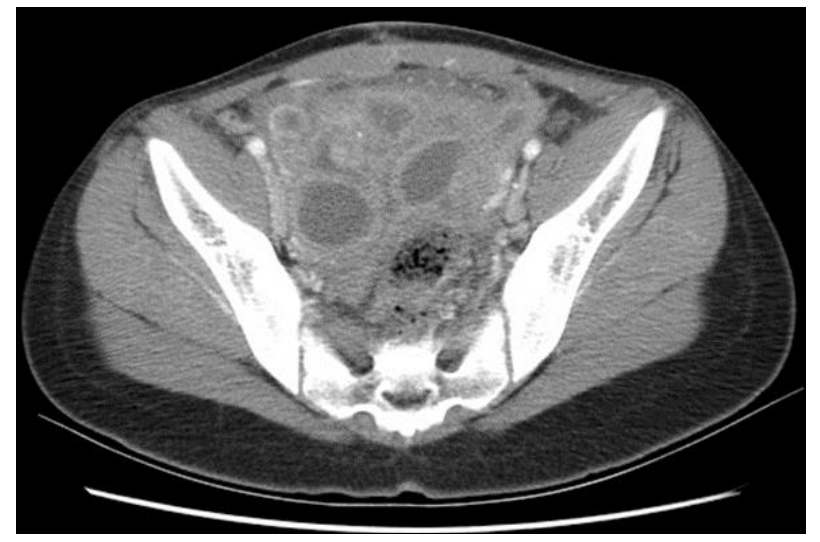

Fig 3: Axial reconstruction, abdominal computed tomography showing large complex cystic lesions in the adnexal region with central hypodensity and peripheral enhancement

\section{Discussion}

According to the European Report about Tuberculosis Surveillance and Monitoring in Europe 2020, children under 15 years accounted for $4.0 \%$ of all new and relapse TB cases, corresponding to a notification rate of 2.5 per 100000 population. Thirty five percent of all TB cases reported in the EU were of foreign origin ${ }^{[9]}$. PTB is a rare form of extrapulmonary tuberculosis that is observed in $0.1-0.7 \%$ of all tuberculosis cases ${ }^{[10]}$.

PTB has been reported primarily in adults between the ages of 35 and 45 years and is extremely rare in children ${ }^{10}$. The diagnosis of PTB requires a high degree of clinical suspicion since the disease is usually insidious and clinical manifestations are nonspecific and laboratory finding can mimic other infectious or malignant diseases ${ }^{[1,11]}$. Because the mortality rate of this disease can reach as high as $51 \%$, one of the most important factors for reducing the mortality rate in children with chronic abdominal complaints is the recognition of this disease ${ }^{[10]}$. Several risk facts have been described in association with PTB as mentioned before. Interestingly, our patient presented none of the reported risk factors for PTB. Although we highlight the immigration from a high risk area. Detailed family histories should be obtained for children with chronic abdominal complaints. One study reported that $60 \%$ of children with PTB have family histories of tuberculosis ${ }^{[10]}$.

Clinical manifestations include abdominal pain $(73 \%)$ and fever $(58 \%)$, which were presented in the case described. Commonly, symptoms have persisted for weeks or months before the diagnosis is achieved. In summary, clinical manifestations of abdominal TB depend on the form of 
disease and may also include weight loss, abdominal distension, hepatomegaly, diarrhea, constipation, abdominal mass, ascites and night sweats ${ }^{[4,10,11]}$.

Among $50 \%-80 \%$ of patients, laboratory tests can demonstrate anemia, thrombocytosis, increased sedimentation rate and C-reactive protein level and the white blood count is usually normal, as seen in this case ${ }^{[1,4}$, $11,12]$.

Pulmonary or pleural involvement has been reported in 50$75 \%$ of patients with PTB. Therefore, chest radiographies to detect the primary focus might be beneficial ${ }^{[10]}$. There was no pulmonary involvement in our patient, who lacked any pulmonary complaints and exhibited normal chest radiography findings.

An elevated serum level of CA-125 is a nonspecific marker of ovarian cancer that can also be elevated in multiple gynaecological and non-gynaecological conditions, including PTB and some authors even suggest that monitoring of CA-125 levels might be beneficial for the evaluation of treatment success. Other non-invasive test that can be adjunctive diagnostic tools to PT include the adenosine deaminase (ADA) levels in ascitic fluid which increase due to stimulation of mycobacterial antigens ${ }^{11}$. Some authors have demonstrated that it is a highly accurate diagnostic test, with a sensitivity and specificity of $100 \%$ and $97 \%[1,11]$. The diagnosis can also be confirmed by Polymerase chain reaction (PCR) of the infected tissues ${ }^{11}$. The utility of PCR varies depending on the tissue type. Sensitivity and specificity are high for pancreatic, hepatic tissue and peritoneal fluid, but intestinal tissue analysis may be associated with false-positive results ${ }^{[4]}$. The yield of ascitic fluid PCR for diagnosis of PTB has not been well established ${ }^{[1,4]}$.

Similar to our case, about $40-85 \%$ of patients with PTB have a positive Mantoux test and this simple, non-invasive test can also provide useful diagnostic information ${ }^{[1,11]}$. Although a positive result from a tuberculin skin test supports the diagnosis, a negative result does not exclude the disease ${ }^{[10]}$. Interferon gamma release assays (IGRAs) are in vitro assays that quantify the level of interferon gamma released by T-lymphocytes in a blood sample after exposure to synthetic TB antigen, which are normally absent from the BCG vaccine ${ }^{[1,12]}$. These assays are dependent on primary versus secondary immunologic response, in which the second response lymphocytes produce a larger amount of interferon gamma relative to the primary response. Therefore, these tests demonstrate a patient's exposure to TB but are unable to distinguish between latent and active infections. Unfortunately, due to the molecular mechanism behind IGRA testing, the frequently immunocompromised states of those with PTB will likely result in reduced test sensitivities/ indeterminate results. For these reasons, IGRAs alone are insufficient in diagnosing PTB $^{12}$. ZiehlNeelsen staining is positive in only $32-35 \%$ and lacks specificity ${ }^{[5]}$. Ziehl-Neelsen stain or Mycobacterium tuberculosis culture require adequate sampling with bacillary lesions, unfortunately peritoneal and genital TB lesions are usually pauci-bacillary ${ }^{[3]}$.

The radiologic features of PT are nonspecific, including ascites, irregular abdominal masses, intra-abdominal lymphadenopathy, omentum and peritoneal thickening and can be detected in other diseases, such as primary malignant tumors, lymphoma and peritonites ${ }^{[1,11]}$.

The gold standard for diagnosis is culture of $\mathrm{Mt}$ in the ascitic fluid or the detection of peritoneal biopsy findings that are compatible with tuberculosis. Indeed, the frequency of a positive ascites culture is known to be less than $50 \%$ [10, ${ }^{11]}$. However, this approach allowed the proper diagnosis in our case.

Laparoscopic or laparotomic examination of the peritoneum followed by histopathology can be used, allowing a more rapid diagnosis of PTB compared to conventional microbiological assays. Among these patients a broad spectrum of finding have been described, including thickened, hyperemic peritoneum with ascites and whitish granular nodules scattered over the peritoneum; ascites and adhesions; and thickened parietal peritoneum with possibly yellowish nodules and cheesy material, along with multiple thickened adhesions ${ }^{[11]}$. In pelvic organs, there may be the following abnormalities: nodular salpingitis, short and swollen tubes with agglutinated fimbriae (patchy salpingitis), unilateral or bilateral hydrosalpinx with retortshaped tubes due to agglutination of fimbriae, or pyosalpinx or caseosalpinx: the tube usually bilateral is distended with caseous material with ovoid white yellow distension of ampulla with poor vascularization ${ }^{[2]}$, as seen in this case. Various types of adhesions may be present in genital TB covering genital organs with or without omentum and intestines ${ }^{[2]}$. The histological lesions of TB show granulomas with multinucleated giant cells, epithelioid cells associated with caseous necrosis. However, these features are not present in the early beginning of the TB ${ }^{[1,}$ 5]. The histopathological analysis also requires adequate sampling especially during laparascopic evaluation. In fact, the diagnosis of genital $\mathrm{TB}$ is challenging as laparoscopy can be very dangerous because there might be many adhesions involving omentum and bowels. In such circumstances, laparotomy remains the more adequate and safer technique ${ }^{[5]}$.

WHO in its guidelines recommended daily therapy of rifampicin $(\mathrm{R})$, isoniazid $(\mathrm{H})$, pyrazinamide $(\mathrm{Z})$ and ethambutol (E) for 2 months followed by daily 4-month therapy of rifampicin $(\mathrm{R})$ and isoniazid $(\mathrm{H})$. Alternatively 2 months intensive phase of RHZE can be daily followed by alternate day combination phase (RH) of 4 months ${ }^{[2,12]}$. Studies examining the efficacy of prolonged dual-agent continuation therapy for 6 months or greater have not shown increased efficacy compared to the standard 4-month regimen. Response to therapy in PTB is usually noted within the first 3 months of treatment and is guided by the resolution of presenting symptoms, such as ascites and normalization of laboratory values ${ }^{[12]}$. Corticosteroids have been used in some studies as an adjuvant therapy during the treatment process of PTB, dating as far back as the 1960's. The theory behind the use of corticosteroids revolves around the idea that they reduce the amount of peritoneal inflammation and fibrosis caused by the infection and ultimately prevent adhesions that can lead to intestinal obstruction. While some modest benefit has been noted in small-scale studies, corticosteroids have not been extensively studied in this capacity and the evidence is conflicting and insufficient to recommend the routine use of steroids in children with abdominal TB and their use in PTB is not regularly recommended $[1,5,12]$. TB can affect the genital organs of young females causing extensive adhesions in pelvis and uterus and irreversible damage to the fallopian tubes and therefore infertility ${ }^{[2,8]}$. Most women with genital TB present with infertility and have poor 
prognosis for fertility in spite of tuberculostatic treatment ${ }^{2}$. Outcome for fertility in female genital TB is only good when tuberculostatic treatment is started in early disease. Infertility is seen in up to $40-50 \%$ cases of genital $\mathrm{TB}^{2}$. Prognosis of PTB depends on comorbidity existence such as cirrhosis, HIV infection, diabetes, mellitus, malignancy, antitumor necrotizing factor (TNF) agents and peritoneal dialysis treatment ${ }^{[1,10]}$. Mortality rate varies between 8 and $50 \%{ }^{[1]}$.

\section{Conclusions}

Peritoneal and genital TB are uncommon in children but, when occurring, they have a high fatality and infertility rate. TBP has no characteristic clinical, laboratory or radiological features that enable its easy diagnosis and constitutes a real diagnostic challenge; therefore, this report highlights the need for a high index of suspicion for an accurate diagnosis. For patients who present with chronic abdominal pain, abdominal distension and fever, particularly those in endemic areas, TBP should be kept in mind during the differential diagnosis of both younger age groups and adults. In our case, the lack of comorbidities or previous history of pulmonary TB contribute for its rarity and challenging diagnostic. In this case, corticosteroids therapy were administered for one month with good clinical response. Corticosteroids given in combination with antituberculous agents may offer benefit by minimizing the inflammation and preventing the postinflammatory fibrosis. However, treatment efficacy is not well demonstrated and there is a need for clinical trials to identify the category of patients most likely to benefit from such therapy.

\section{References}

1. Maia EB, Machado M, Januario G, Piedade C, Pinto A, Flores MM. Abdominal tuberculosis in children. Acta Pediatr Port 2015;46:42-45.

2. Sharma JB. Current diagnosis and management of female genital tuberculosis. J Obstet Gynecol India 2015;65(6):362-71.

3. Efared B, Sidibé IS, Erregad F, Hammas N, Chbani L, Fatemi HE. Female genital tuberculosis: a clinicopathological report of 13 cases. J Surg Case Rep 2019;3:1-4.

4. Jurado LF, Pinzón B, Rosa-Noriega ZR, Matijasevic E, López-Panqueva RP. Peritoneal tuberculosis in a health-care worker, radio-pathological assessment and diagnosis, a case report. Radiology of Infectious Diseases 2019;6:163-169.

5. Delisle M, Seguin J, Zeilinski D, Moore D. Paediatric abdominal tuberculosis in developed countries: case series and literature review. Arch Dis Child 2016;101(3):253-8.

6. Malik R, Srivastava A, Yachha S, Poddar U, Lal R. Childhood abdominal tuberculosis: disease patterns, diagnosis, and drug resistance. Indian J Gastroenterol 2015;34(6):418-25.

7. Gascón J, Acién P. Large bilateral tubercular pyosalpinx in a young woman with genitourinary malformation: a case report. J Med Case Rep 2014;8:176.

8. Mehershahi S, Haider A, Fortuzi K, Shaikh D, Patel H. Abdominal ascites of unknown origin: diagnostic accuracy of adenosine deaminase for tuberculous peritonitis. Case Rep Gastroenterol 2021;15(1):418-
423.

9. European Centre for Disease Prevention and Control/WHO Regional Office for Europe. Tuberculosis Surveillance and Monitoring in Europe 2020. https://www.ecdc.europa.eu/en/publicationsdata/tuberculosis-surveillance-and-monitoring-europe2020-2018-data.

10. Avcu G, Sensoy G, Karli A, Caltepe G, Sullu Y, Belet $\mathrm{N}$ et al. A case of tuberculous peritonitis in childhood. J Infect Public Health 2015;8(4):369-72.

11. Tomás C, Lopez B, Couto S, Cordeiro A, Saraiva J. Peritoneal tuberculosis: diagnostic challenge for the gynecologist. Acta Obstet Ginecol Port 2017;11(1):6163.

12. Wu DC, Averbukh LD, Wu GY. Diagnostic and therapeutic strategies for peritoneal tuberculosis: a review. J Clin Transl Hepatol 2019;7(2):140-148. 